\title{
PREVALÊNCIA DE SINTOMAS GRIPAIS NA PANDEMIA POR SARS-COV-2 EM IDOSOS DE SERVIÇO REFERÊNCIA NA AMAZÔNIA
}

Ana Carla Costa Azevedo; Universidade do Estado do Pará (UEPA); carlaazevedo04@gmail.com Andrea Marcela dos Santos Lopes; Universidade do Estado do Pará (UEPA); am.llopess@gmail.com

Davi Gabriel Barbosa; Universidade do Estado do Pará (UEPA); barbosagabrieldavi@gmail.com

Lucas Matheus da Silva Castro; Universidade do Estado do Pará (UEPA); castrolums@gmail.com Paula Gabriela Nascimento Gonçalves; Universidade do Estado do Pará (UEPA); paulagabimed@gmail.com Rayssa Moreira Lacerda; Universidade do Estado do Pará (UEPA); rayssaalacerdas@gmail.com Murilo da Silva Rodrigues; Universidade do Estado do Pará (UEPA); murilorodrigues7@outlook.com Cynthia Cyllene de Oliveira Charone; Grupo Cynthia Charone (GCC); cynthiacharone@yahoo.com.br

\section{RESUMO}

Introdução: A população idosa mereceu importante atenção durante a pandemia do Sars-coV-2, sobretudo pelo fato desta faixa etária apresentar maior susceptibilidade às complicações e aos óbitos, visto que a imunossenescência aumenta a vulnerabilidade a doenças infectocontagiosas, e pelas comorbidades associadas. Neste cenário, ressalta-se que os sintomas gripais são considerados sinais de alerta para Covid-19, especialmente tosse seca, febre, falta de ar e confusão mental, merecendo, portanto, orientação e acompanhamento médico. Objetivo: Avaliar a prevalência de sintomas gripais em um grupo de idosos no período de pandemia do novo coronavírus. Método: Estudo transversal, descritivo, com dados de 799 idosos entrevistados via questionário avaliativo, elaborado e conduzido pelo Grupo Cynthia Charone, entre idosos ativos no programa de envelhecimento saudável. A coleta de dados foi realizada via telemonitoramento, entre maio e junho de 2020 a partir da avaliação das fichas on-line preenchidas. Todos os participantes concordaram em participar do estudo a partir da assinatura voluntaria do Termo de Consentimento Livre e Esclarecido (TCLE) e aprovação do CEP. Resultados: dos 799 idosos avaliados 11,7\% referiram ter apresentado tosse, $8,2 \%$ alegaram coriza, $5 \%$ relataram fadiga, $4,1 \%$ apresentaram falta de ar, $4 \%$ alegaram anosmia e/ou ageusia, 3,7\% referiram febre, 3,3\% alegaram dor de garganta, $2,7 \%$ relataram diarreia e 1,7\% referiram náusea ou vômito. Conclusão: Os sintomas gripais mais prevalentes entre os idosos acompanhados pelo GCC, durante a pandemia do coronavírus foram: tosse, coriza e fadiga e o seu entendimento é demasiadamente importante frente ao contexto pandêmico e o risco que os idosos apresentam.

Palavras-chave: Tosse; Coriza; Infecções por coronavírus; Assistência integral à saúde do idoso. 